\title{
Therapeutic Effect of Abelmoschus manihot on Type 2 Diabetic Nonproliferative Retinopathy and the Involvement of VEGF
}

\author{
Yue Zhao ${ }^{1},{ }^{1}$ Xu Yu, ${ }^{1}$ Yan Lou, ${ }^{1}$ Xinyi Sun, ${ }^{1}$ Boyu Zhu, ${ }^{1}$ Weilong Xu, ${ }^{1}$ Lei Zhou, ${ }^{1}$ Hao Wu, \\ Qingzi Jin, ${ }^{2}$ Heng Wang, ${ }^{3}$ Jianjiang Shen, ${ }^{3}$ Jiangyi Yu $\mathbb{D}^{1},{ }^{1}$ and Xiaofei An $\mathbb{D}^{1}$ \\ ${ }^{1}$ Department of Endocrinology, Affiliated Hospital of Nanjing University of Chinese Medicine, Nanjing, China \\ ${ }^{2}$ Department of Ophthalmology, Affiliated Hospital of Nanjing University of Chinese Medicine, Nanjing, China \\ ${ }^{3}$ Department of Laboratory, Affiliated Hospital of Nanjing University of Chinese Medicine, Nanjing, China
}

Correspondence should be addressed to Xiaofei An; anxiaofeitcm@126.com

Received 13 December 2019; Revised 23 March 2020; Accepted 2 April 2020; Published 30 April 2020

Academic Editor: Wenyi Kang

Copyright (c) 2020 Yue Zhao et al. This is an open access article distributed under the Creative Commons Attribution License, which permits unrestricted use, distribution, and reproduction in any medium, provided the original work is properly cited.

Objective. To evaluate the efficacy of Abelmoschus manihot in treating type 2 diabetic nonproliferative retinopathy. Methods. It was a randomized controlled clinical trial. The recruited eighty subjects with type 2 diabetic nonproliferative retinopathy were randomly divided into treatment group and control group. The two groups received basic treatments including control of blood glucose, blood pressure and blood lipid, management of diet, exercise and health education, and monitoring of relevant indicators. Additionally, the treatment group was given oral administration of Abelmoschus manihot. All subjects were followed up on monthly basis for consecutive six months. The related parameters including diabetic retinopathy (DR) incidence rates, "Early Treatment Diabetic Retinopathy Study" (ETDRS) vision scores, retinal thicknesses in macular region, serum vascular endothelial growth factor (VEGF) levels, and biochemical indicators of both groups before and after treatment were accurately collected and statistically analyzed. Results. There were no significant differences of DR severity levels, ETDRS vision scores, macular retinal thicknesses such as cube average thickness (CAT), central subfield thickness (CST), and cube volume (CV), and serum VEGF levels between two groups before treatment. Meanwhile, there were no significant differences of demographic characteristics, case terminations, blood glucose, blood lipid, blood pressure, biochemical indicators of hepatorenal function, hypoglycemic drugs, hypotensive drugs, and other basic treatments between two groups during six months treatment. The present study suggested that the remission rate of DR and the ETDRS vision score in the treatment group were significantly higher than those of the control group (remission rate: $25.4 \%$ vs $9.3 \%, P=0.01$; ETDRS score: $78(72,82)$ vs $72(67,80), P=0.0002)$ while the progression rate of DR in the treatment group was significantly lower than that of the control group (progression rate: $4.2 \%$ vs $18.7 \%, P=0.007$ ) after six months treatment. In addition, the CAT, CST, CV, and serum VEGF levels of the treatment group were significantly improved after the treatment (CAT: $286(278,302)$ vs $282(270,295) \mu \mathrm{m}, P<0.0001$; CST: $251(239,274)$ vs $248(235,265) \mu \mathrm{m}, P<0.0001$; CV: $10.3(10.0,10.9)$ vs $10.1(9.7,10.6) \mathrm{mm}^{3}, P<0.0001$; VEGF: $0.21(0.14,0.58)$ vs $\left.0.16(0.10,0.23) \mathrm{ng} / \mathrm{ml}, P=0.0026\right)$, while there were no significant differences of the control group before and after treatment (CAT: $287(279,294)$ vs $287(279,295) \mu \mathrm{m}$, $P=0.27$; CST: $250(240,266)$ vs $252(238,266) \mu \mathrm{m}, P=0.72$; CV: $10.4(10.1,10.6)$ vs $10.4(10.1,10.7) \mathrm{mm}^{3}, P=0.53$; VEGF: 0.21 $(0.13,0.66)$ vs $0.23(0.12,0.64) \mathrm{ng} / \mathrm{ml}, P=0.85)$. Conclusion. The study offered the novel evidence for the therapeutic effect of Abelmoschus manihot on type 2 diabetic nonproliferative retinopathy, which was associated with improved VEGF. This trial is registered with ChiCTR1800019292.

\section{Introduction}

As one of the major microvascular complications of diabetes, diabetic retinopathy (DR) is regarded as the leading cause of vision loss in middle-aged and elderly people all over the world [1]. A meta-analysis including 35 studies and 22,896 diabetes patients showed that the incidence of DR in diabetes was about $34.6 \%$, among which the proliferative diabetic retinopathy (PDR) took up 6.96\%, the diabetic macular edema (DME) about $6.81 \%$, and the 
vision-threatening diabetic retinopathy (VTDR) about $10.2 \%$ [2]. With the extension of diabetic duration, the prevalence of DR keeps increasing [3] and the global medical cost required for the prevention and treatment of $\mathrm{DR}$ is also rising [1]. DR is becoming increasingly harmful to human health and burdened to the medical care system. Therefore, it is very meaningful to explore effective agents or treatment for preventing the progression of DR.

According to Diabetic Retinopathy Preferred Practice Pattern Guideline (Version 2017) issued by American Academy of Ophthalmology, strict control of blood glucose, blood pressure, blood lipid, and other metabolic disorders is taken as the main method of secondary prevention of DR. The antivascular endothelial growth factor (VEGF) therapy, panretinal photocoagulation, and vitrectomy could be adopted when severe nonproliferative diabetic retinopathy (NPDR), PDR, or clinically significant macular edema (CSME) occurs [4]. Several studies indicated that intravitreal dexamethasone (DEX) implant was effective for long term and safe to DME and diabetic tractional retinal detachment [5-8]. Additionally, the improvement of vision and macular retinal thickness by DEX was in both eyes that were treatment-naive and eyes refractory to anti-VEGF treatment [9]. However, all these surgical treatments are required to be operated by experienced ophthalmologists. For endocrinologists and general physicians who are responsible for DR management, it is essential to select the appropriate methods to prevent further progression at the early stage of DR. Although the first-line treatment including the control of blood glucose, blood pressure, and blood lipids has been adopted in current clinical practice, the development of DR still remains not to be mitigated and irreversible.

The recent study indicated the validity of the commonly used anti-DR drugs such as alprostadil, antioxidants, antithrombotics, and protein kinase-C inhibitors was lack of evidence from large-sample clinical research [10]. It seems that searching for some complementary and alternative agents from traditional Chinese medicine for DR may partly alleviate this dilemma. The studies showed that some herbal medicines reduced the apoptosis of retinal pericytes (RPCs), maintained the function of retinal endothelial cells (RECs), and inhibited retinal neovascularization by improving the oxidative stress and inflammatory state of DR [11]. The present six-month prospective clinical study was performed to investigate the clinical efficacy and possible mechanism of Abelmoschus manihot, a traditional Chinese medicine in treating type 2 diabetic nonproliferative retinopathy [12].

\section{Methods}

2.1. Study Design. Eighty eligible type 2 diabetic patients with nonproliferative retinopathy who met the following criteria were recruited in this randomized controlled clinical trial. Inclusion criteria include the following: (1) type 2 diabetes with nonproliferative retinopathy diagnosed by fundus photography or fluorescein angiography; (2) age 18 70 years old, male or female; (3) in the last 12 weeks, the highest blood glucose $<16.7 \mathrm{mmol} / \mathrm{L}$, blood pressure $\leq 140 /$ $90 \mathrm{mmHg}$, and body mass index (BMI) $<30 \mathrm{~kg} / \mathrm{m}^{2}$; (4) completely understanding and signing informed consent. Exclusion criteria were as follows: (1) acute diabetic complications in last 4 weeks such as diabetic ketoacidosis or diabetic nonketogenic hyperosmolar syndrome; (2) serious chronic diabetic complications such as diabetic nephropathy with nephrotic syndrome, severe diabetic peripheral neuropathy, or diabetic lower-extremity artery occlusion; (3) retinal vascular occlusion, uveitis, age-related macular degeneration, hypertensive retinopathy, or other diseases leading to macular edema; (4) severe cataract affecting vision test or glaucoma limiting mydriasis; (5) serious systemic diseases such as cancer, severe inflammation, AIDS, heart failure, end-stage renal disease, respiratory failure, and hematologic or mental disorder; (6) pregnant or lactating women, allergic constitution, or poor adherence.

The randomized method in this study was block randomization with 4 subjects in one block and 20 blocks in total. The random arrangement (random coding table) for 80 subjects was generated by SAS statistical software. All subjects were assigned to a number from 1 to 80 according to the chronological order of enrollment and then randomly divided into the treatment group and control group, each with 40 cases.

All subjects were enrolled to receive comprehensive medical history collection, physical examination, test of blood glucose, blood lipid and other biochemical indicators, fundus photography, "Early Treatment Diabetic Retinopathy Study" (ETDRS) vision test, optical coherence tomography (OCT) examination, and serum VEGF assay. Both groups were given basic treatments including control of blood glucose, blood pressure and blood lipid, management of diet, exercise and health education, and monitoring of relevant indicators according to "China Guideline for Type 2 Diabetes." The treatment group was additionally given oral administration of Abelmoschus manihot (Jiahua tablets) with 1.8 grams per time and three times every day. Jiahua tablets, as the semiextract tablets of Abelmoschus manihot, were produced by the Affiliated Hospital of Nanjing University of Chinese Medicine with production approval code Z04000511.

All subjects were followed up on monthly basis for consecutive six months. The related indicators including DR incidence rates, ETDRS vision scores, macular retinal thicknesses, and serum VEGF levels of both groups before and after treatment were tested and compared to evaluate the efficacy of Jiahua tablets in treating DR. The research procedure was shown in Figure 1.

2.2. Main Observation Items and Testing Instruments. The NPDR severity levels (mild/moderate/severe) were assessed by the fundus camera (Kowa Nonmyd 7). The vision scores were evaluated by using the ETDRS vision chart. The retinal thicknesses in macular region were measured by the OCT instrument (Cirrus HD-OCT 5000, Carl Zeiss Meditec). The serum VEGF levels were assayed with the human VEGF-A ELISA kit (NJJCBIO Company, Nanjing, China) by the ELISA microplate reader (Bio Tek ELx800). The biochemical indicators including fasting blood glucose (FBG), postprandial blood glucose (PBG), glycosylated hemoglobin A1c 


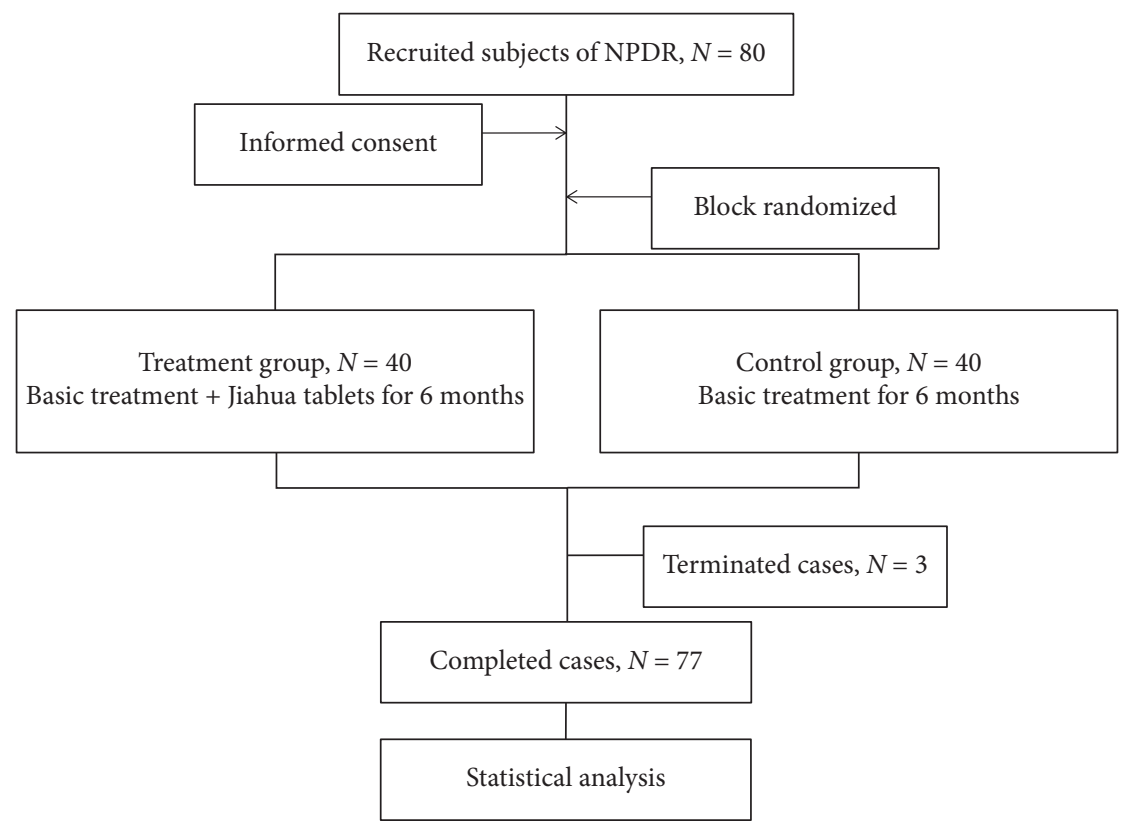

FIgURE 1: Study design and procedure.

(HbA1c), total cholesterol (TC), triglyceride (TG), highdensity lipoprotein cholesterol (HDL), low-density lipoprotein cholesterol (LDL), alanine aminotransferase (ALT), aspartate aminotransferase (AST), blood urea nitrogen (BUN), serum creatinine ( $\mathrm{Scr}$ ), and estimated glomerular filtration rate (eGFR) were tested by the automatic chemistry analyzer (Beckman Coulter AU5800). The demographic characteristics and vital signs were also collected.

2.3. Relevant Attentions in the Study. When an adverse reaction of some subject was detected, the chief researcher needed to decide how to conduct further treatment and whether to discontinue the study of this subject. When a severe adverse event occurred, the research leader should immediately take necessary actions to protect the safety of the subject and, meanwhile, fill out the "severe adverse events report form" and report to the ethics committee of research institute within 24 hours to ensure that the reporting process meets all legal and regulatory requirements.

Part of subjects was considered to be terminated cases for some reasons as follows. Severe adverse events or other adverse reactions occurred; the study plan was obviously deviated (e.g., poor compliance of medication), and researchers thought it difficult to evaluate drugs' effect; subjects did not want to continue and applied for withdrawing from the study.

As for quality control and data management, all researchers needed to pass good clinical practice training and strictly follow the trial protocol in the study. Meanwhile, relevant laboratory technicians should comply with the standard operation procedure (SOP) of index testing, and the testing results should be traceable. The repetitive tests of fundus photography, ETDRS vision, OCT levels, and other indicators were performed by the same operators with same instruments according to the SOP. All subjects' data collected in the study were recorded in case report forms by researchers according to the SOP and checked regularly by quality controllers to ensure the authenticity, timeliness, accuracy, and integrity.

The enrolled subjects were randomly divided into the treatment group and control group according to the random coding table. All patients' indicators including biochemical indicators, blood pressure, DR severity, ETDRS score, macular retinal thickness, and serum VEGF were tested by special assessors of this study. However, the assessors were masked to grouping details. The intraclass correlation coefficients (ICC) of these indicators were calculated with the two-way random model and single absolute agreement type by SPSS 22.0. The estimated ICC values ranged from $0.85 \sim 0.92$. The interobserver reliability and test-retest reliability of indicators were considered to be valid according to the statistical principle.

2.4. Ethics. This trial was approved by the Ethics Committee of Affiliated Hospital of Nanjing University of Chinese Medicine (approval number: 2018NL-104-03) and registered the Chinese Clinical Trial Registry (registration number: ChiCTR1800019292). All subjects were enrolled in this study with signed informed consents which explained in detail the study's objective and procedure, possible benefits and risks, rights, and obligations.

2.5. Statistical Analysis. Values conforming to normal distribution were expressed as mean \pm standard deviation $(\bar{x} \pm \mathrm{SD})$, while those not conforming to normal distribution were expressed as median with interquartile range $(M(Q 1$, Q3)). The measurement data were analyzed by $t$ test (paired $t$ test) or rank-sum test (paired rank-sum test) according to 
whether they conformed to the normal distribution, while the categorical data were mainly analyzed by chi-squared test or Fisher's exact test. It was two-sided test, and " $P \leq 0.05$ " was considered to be statistically significant. The statistic software was SAS 9.4 (SAS Institute Inc, Cary, North Carolina).

\section{Results}

(1) As shown in Table 1, there were no significant differences of demographic characteristics including sex, age, DM duration, and BMI between the two groups.

(2) Three cases subjects were terminated during the sixmonth treatment period including one case with automatic quit, one case with poor compliance in the treatment group, and another case with automatic quit in the control group. There were no significant differences in case terminations between the two groups as shown in Table 2.

(3) There were no significant differences of blood glucose, blood lipid, blood pressure, and biochemical indicators of liver and kidney function between two groups before and after treatment as shown in Table 3.

(4) Before treatment, eight unilateral eyes were excluded for observation including four with severe cataract and one with blindness due to trauma in the treatment group and two with severe cataract and one with severe nondiabetic macular disease in the control group. After six months' treatment, two cases (4 eyes) in the treatment group and one case ( 2 eyes) in the control group were terminated and excluded for observation.

There were 31 eyes with mild NPDR, 31 eyes with moderate NPDR, and 13 eyes with severe NPDR in the treatment group, while 31 eyes with mild NPDR, 36 eyes with moderate NPDR and 10 eyes with severe NPDR in the control group before treatment. There was no significant difference of NPDR severity between two groups before treatment as shown in Table 4.

However, after six months' treatment, there were 42 eyes with mild NPDR, 25 eyes with moderate NPDR, and 4 eyes with severe NPDR in the treatment group, while 27 eyes with mild NPDR, 35 eyes with moderate NPDR, and 13 eyes with severe NPDR in the control group. There was a significant difference of NPDR severity between two groups after treatment $(P=0.008)$ as shown in Table 4 .

Additionally, the remission rate of NPDR severity in the treatment group was significantly higher than that of the control group $(25.4 \%$ vs $9.3 \%, P=0.01)$ and the progression rate of NPDR severity in the treatment group was significantly lower than that of the control group $(4.2 \%$ vs $18.7 \%, P=0.007)$ as shown in Table 5 . The typical changes of fundus photographs before and after treatment in the two groups are shown in Figure 2.

(5) There was no significant difference of ETDRS vision score between two groups before treatment; however, the ETDRS vision score in the treatment group was significantly higher than that of the control group after six months' treatment $(78(72,82)$ vs 72 $(67,80), P<0.001)$ as shown in Table 6 .

(6) There were no significant differences of cube average thickness (CAT), central subfield thickness (CST), and cube volume $(\mathrm{CV})$ in macular region between two groups before treatment as shown in Table 7.

As shown in Figure 3, the treatment group's CAT, CST, and CV were significantly lower after treatment (CAT: $286(278,302)$ vs $282(270,295) \mu \mathrm{m}$, $P<0.0001$; CST: $251(239,274)$ vs $248(235,265) \mu \mathrm{m}$, $P<0.0001 ; C V: 10.3(10.0,10.9)$ vs $10.1(9.7,10.6)$ $\left.\mathrm{mm}^{3}, P<0.0001\right)$, while there were no significant differences of control group's CAT, CST and CV before and after treatment (CAT: $287(279,294)$ vs $287(279,295) \mu \mathrm{m}, P=0.27$; CST: $250(240,266)$ vs $252(238,266) \mu \mathrm{m}, P=0.72$; CV: $10.4(10.1,10.6)$ vs $\left.10.4(10.1,10.7) \mathrm{mm}^{3}, P=0.53\right)$. The typical changes of OCT images and parameters before and after treatment in the two groups are shown in Figure 4.

(7) There were no significant differences of serum VEGF levels between two groups before treatment; however, the treatment group's VEGF level was significantly lower than that of the control group after six months' treatment $(0.16(0.10,0.23)$ vs $0.23(0.12$, $0.64), P=0.02$ ) as shown in Table 8 . The serum VEGF levels of the treatment group were significantly lower after six months' treatment $(0.21$ ( 0.14 , $0.58)$ vs $0.16(0.10,0.23) \mathrm{ng} / \mathrm{ml}, P=0.0026)$; however, there was no significant difference of the control group's VEGF levels before and after treatment $(0.21(0.13,0.66)$ vs $0.23(0.12,0.64) \mathrm{ng} / \mathrm{ml}$, $P=0.85)$ as shown in Figure 3.

(8) There were no significant differences of hypoglycemic drugs, hypotensive drugs, and other basic treatments between two groups during six months' treatment as shown in Table 9.

\section{Discussion}

As we know, Youyou Tu, the Nobel Prize winner, was inspired by the ancient medical document and discovered artemisinin by changing the drug extraction method. We could seek inspiration from traditional theories and experiences from the valuable resource of Chinese medicine. According to Chinese medicine literature, "the liver and kidney share the same origin and the eye and kidney share the same treatment." The main functions of Abelmoschus manihot are "clearing heat and dampness, promoting blood circulation for removing obstruction in collaterals, and reducing swelling and detoxifying" [13]. Meanwhile, Abelmoschus manihot are effectively and commonly used for diabetic nephropathy [14-16]. These theories and 
TABLE 1: Comparisons of demographic characteristics between two groups.

\begin{tabular}{lccc}
\hline & T group $(n=40)$ & C group $(n=40)$ & $P$ \\
\hline Sex (male/female) & $23 / 17$ & $27 / 13$ & 0.36 \\
Age (years) & $61(52,64)$ & $55(50,63)$ & 0.16 \\
DM duration (years) & $10(5,15)$ & $8(5,13)$ & 0.28 \\
BMI $\left(\mathrm{kg} / \mathrm{m}^{2}\right)$ & $25.9 \pm 3.6$ & $26.4 \pm 3.3$ & 0.48 \\
\hline
\end{tabular}

T: treatment; C: control; BMI: body mass index.

TABLe 2: Comparisons of case terminations between two groups after treatment.

\begin{tabular}{lccc}
\hline & T group $(n=38)$ & C group $(n=39)$ & $P$ \\
\hline Total terminated case & $2(5.0 \%)$ & $1(2.5 \%)$ & \\
Causes of termination & & 1 & 1.00 \\
Automatic quit & 1 & 0 & 1.00 \\
Poor compliance & 1 & 0 & \\
Drug reaction & 0 & 0 & \\
Adverse events & 0 & & \\
\hline
\end{tabular}

T: treatment; C: control.

TABLE 3: Comparisons of biochemical indicators and blood pressure between two groups before and after treatment.

\begin{tabular}{|c|c|c|c|c|c|c|}
\hline & \multicolumn{2}{|c|}{ T group } & \multicolumn{2}{|c|}{ C group } & \multirow{2}{*}{$P_{1}$} & \multirow{2}{*}{$P_{2}$} \\
\hline & Before $(n=40)$ & After $(n=38)$ & Before $(n=40)$ & After $(n=39)$ & & \\
\hline $\mathrm{FBG}(\mathrm{mmol} / \mathrm{L})$ & $8.7 \pm 2.6$ & $7.8 \pm 1.9$ & $8.2 \pm 2.9$ & $8.1 \pm 2.2$ & 0.37 & 0.47 \\
\hline PBG (mmol/L) & $12.7 \pm 2.2$ & $10.5 \pm 2.1$ & $11.9 \pm 3.0$ & $10.8 \pm 2.5$ & 0.19 & 0.49 \\
\hline HbAlc (\%) & $8.8 \pm 1.7$ & $7.8 \pm 1.8$ & $9.1 \pm 2.4$ & $7.8 \pm 1.4$ & 0.51 & 0.95 \\
\hline TC (mmol/L) & $4.3 \pm 1.0$ & $4.3 \pm 1.0$ & $4.1 \pm 1.0$ & $4.2 \pm 1.0$ & 0.41 & 0.53 \\
\hline TG $(\mathrm{mmol} / \mathrm{L})$ & $1.7(1.1,2.4)$ & $1.5(1.2,2.2)$ & $1.6(1.1,2.5)$ & $1.7(1.1,2.2)$ & 0.77 & 0.74 \\
\hline $\mathrm{HDL}(\mathrm{mmol} / \mathrm{L})$ & $1.2 \pm 0.3$ & $1.4 \pm 0.3$ & $1.2 \pm 0.3$ & $1.3 \pm 0.3$ & 0.74 & 0.13 \\
\hline $\mathrm{LDL}(\mathrm{mmol} / \mathrm{L})$ & $2.7 \pm 0.8$ & $2.9 \pm 0.9$ & $2.5 \pm 0.8$ & $2.8 \pm 1.0$ & 0.48 & 0.70 \\
\hline $\operatorname{ALT}(\mathrm{U} / \mathrm{L})$ & $26(18,42)$ & $29(20,38)$ & $30(19,40)$ & $29(21,35)$ & 0.60 & 0.77 \\
\hline AST (U/L) & $22(17,27)$ & $22(19,28)$ & $22(18,28)$ & $23(20,29)$ & 0.42 & 0.56 \\
\hline $\mathrm{BUN}(\mathrm{mmol} / \mathrm{L})$ & $6.7 \pm 2.0$ & $6.5 \pm 1.5$ & $6.2 \pm 1.2$ & $6.0 \pm 1.3$ & 0.22 & 0.14 \\
\hline Scr (umol/L) & $67(60,81)$ & $71(61,80)$ & $68(57,75)$ & $70(58,76)$ & 0.61 & 0.50 \\
\hline eGFR (mL/min) & $97.5 \pm 25.7$ & $98.3 \pm 22.1$ & $99.8 \pm 17.2$ & $98.8 \pm 16.6$ & 0.64 & 0.91 \\
\hline $\mathrm{SBP}(\mathrm{mmHg})$ & $128 \pm 12$ & $129 \pm 8$ & $126 \pm 10$ & $130 \pm 8$ & 0.32 & 0.93 \\
\hline DBP (mmHg) & $75 \pm 10$ & $77 \pm 5$ & $76 \pm 8$ & $78 \pm 6$ & 0.54 & 0.31 \\
\hline
\end{tabular}

T: treatment; C: control; SBP: systolic blood pressure; DBP: diastolic blood pressure; $\mathrm{P}_{1}$ : T group vs $\mathrm{C}$ group before treatment; $\mathrm{P}_{2}$ : T group vs $\mathrm{C}$ group after treatment.

TABLE 4: Comparisons of NPDR severity between two groups before and after treatment.

\begin{tabular}{lccccc}
\hline & \multicolumn{2}{c}{ T group } & \multicolumn{2}{c}{ C group } & $P_{1}$ \\
& Before $(n=75)$ & After $(n=71)$ & Before $(n=77)$ & After $(n=75)$ & $27(36.0 \%)$ \\
Mild & $31(41.3 \%)$ & $42(59.2 \%)$ & $31(40.2 \%)$ & $35(46.7 \%)$ & 0.69 \\
Moderate & $31(41.3 \%)$ & $25(35.2 \%)$ & $36(46.8 \%)$ & $13(17.3 \%)$ & $0.008^{*}$ \\
Severe & $13(17.4 \%)$ & $4(5.6 \%)$ & $10(13.0 \%)$ & \\
\hline
\end{tabular}

T: treatment; $\mathrm{C}$ : control; $\mathrm{P}_{1}$ : T group vs $\mathrm{C}$ group before treatment; $\mathrm{P}_{2}$ : T group vs $\mathrm{C}$ group after treatment, ${ }^{*} \mathrm{P} \leq 0.05$.

experiences induced our hypothesis of applying Abelmoschus manihot for DR treatment. The pharmacological study showed that total flavone of Abelmoschus (TFA) is the major active component of Abelmoschus manihot. TFA is mainly composed of several flavonoids including quercetin, hyperoside, and isoquercitrin $[17,18]$. It was shown that quercetin has a dose-dependent effect in inhibiting proliferation of rhesus macaque choroid-retinal endothelial cells [19] and reducing apoptosis of retinal ganglion cell layer and whole retinal vessels in diabetic rats $[20,21]$. Meanwhile, the previous study has confirmed that quercetin could significantly inhibit the expression of retinal VEGF in diabetic rats [22]. High glucose induced the VEGF overexpression in the retina, which could damage the 
TABLE 5: Comparisons of variations of NPDR severity between two groups after treatment.

\begin{tabular}{lccc}
\hline & T group $(n=71)$ & C group $(n=75)$ & $P$ \\
\hline Improved & $18(25.4 \%)$ & $7(9.3 \%)$ & $0.01^{*}$ \\
Maintained & $50(70.4 \%)$ & $54(72.0 \%)$ & 0.83 \\
Aggravated & $3(4.2 \%)$ & $14(18.7 \%)$ & $0.007^{*}$ \\
\hline
\end{tabular}

T: treatment; C: control; ${ }^{*} P \leq 0.05$.
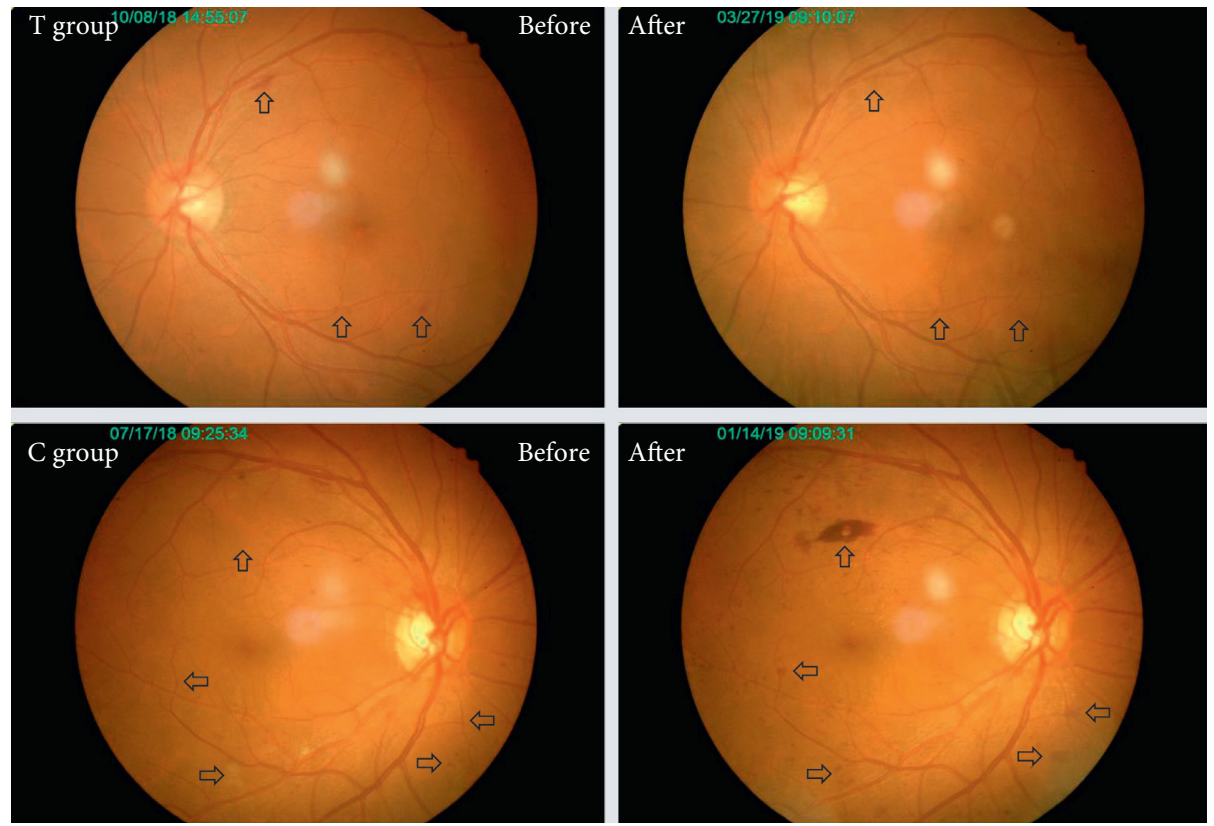

Figure 2: The typical changes of fundus photographs before and after treatment in the two groups. As indicated by the arrows in the figure, the retinal hemorrhage and exudation in the treatment group were significantly reduced after six months' treatment. In contrast, the retinal hemorrhage and exudation in the control group were significantly increased. T: treatment; C: control.

TABLE 6: Comparisons of ETDRS vision score between two groups before and after treatment.

\begin{tabular}{lccccrr}
\hline & \multicolumn{2}{c}{ T group } & \multicolumn{2}{c}{ C group } & $P_{1}$ \\
& Before $(n=75)$ & After $(n=71)$ & Before $(n=77)$ & After $(n=75)$ & $P_{2}$ \\
\hline ETDRS score & $73(67,79)$ & $78(72,82)$ & $74(68,80)$ & $72(67,80)$ & 0.26 \\
\hline
\end{tabular}

T: treatment; C: control; P1: T group vs $\mathrm{C}$ group before treatment; $\mathrm{P} 2$ : T group vs $\mathrm{C}$ group after treatment, ${ }^{*} \mathrm{P} \leq 0.05$.

TABLE 7: Comparisons of macular thicknesses between two groups before treatment.

\begin{tabular}{lccc}
\hline & T group $(n=75)$ & C group $(n=77)$ & $P$ \\
\hline CAT (um) & $286(278,302)$ & $287(279,294)$ & 0.67 \\
CST $($ um) & $251(239,274)$ & $250(240,266)$ & 0.78 \\
CV $\left(\mathrm{mm}^{3}\right)$ & $10.3(10.0,10.9)$ & $10.4(10.1,10.6)$ & 0.62 \\
\hline
\end{tabular}

T: treatment; C: control.

blood-retinal barrier (BRB) and result in pathogenesis of DR through multiple pathways. VEGF could accelerate the apoptosis of retinal pericytes (RPCs) and abnormal proliferation of retinal endothelial cells (RECs) $[23,24]$ and destroy intercellular tight-junctions proteins as occludin and claudin $[25,26]$. It also could induce the increased expression of intercellular adhesion molecule-1 [27], inhibit the antagonistic effect of pigment epithelium-derived factor
(PEDF) on VEGF [28], and promote the cell swallowing and endothelial cell perforation. Therefore, we aimed to explore the possible therapeutic effect of Abelmoschus manihot (Jiahua tablets) on treating NPDR in the present study.

The fundus photograph was comprehensively used in DR screening because of the convenient operation for physicians and easy cooperation for patients. But, there was disadvantage of unclear images in patients with severe 


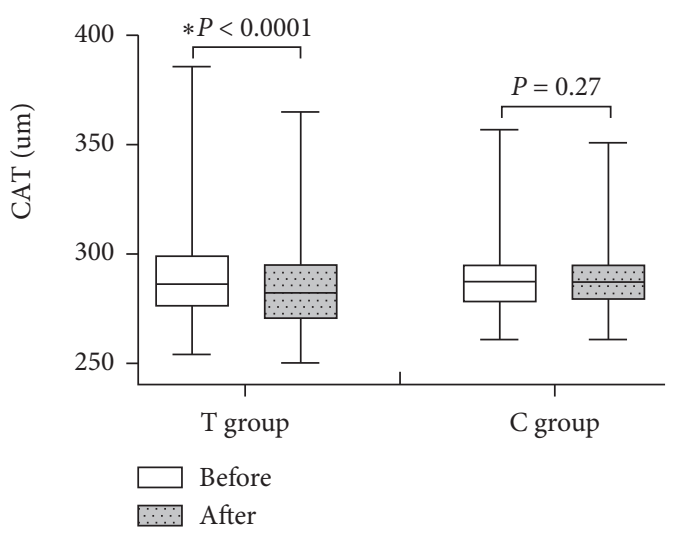

(a)

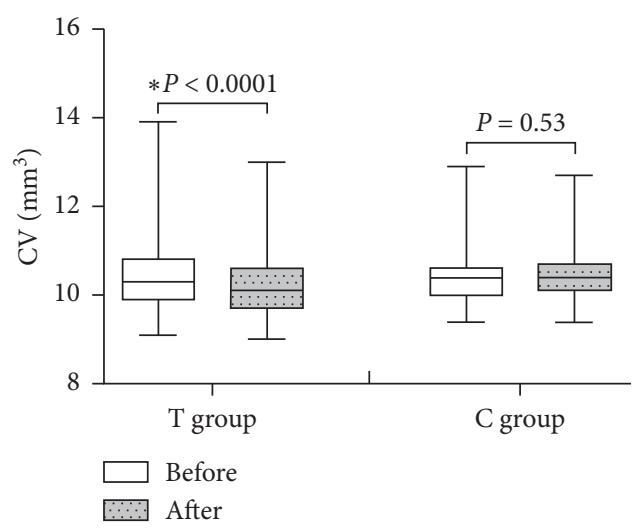

(c)

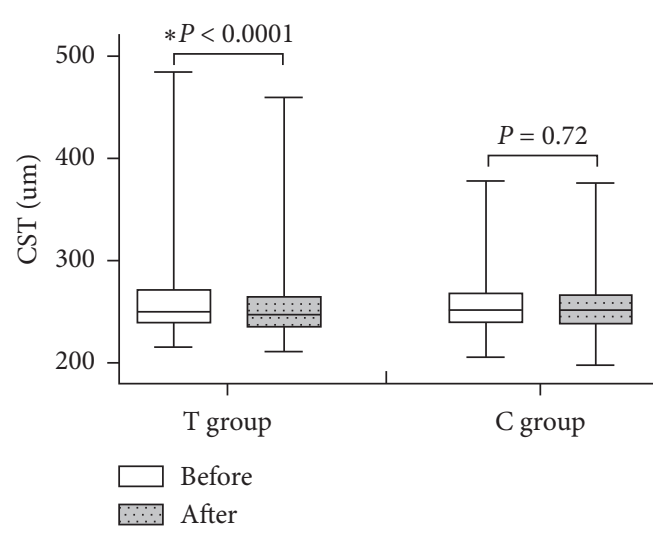

(b)

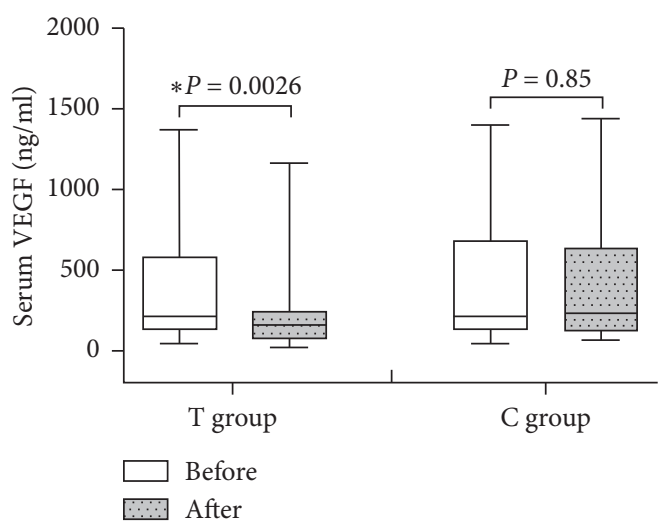

(d)

FIgURE 3: Comparisons of CAT, CST, CV, and VEGF of two groups before and after treatment. The treatment group's CAT, CST, and CV levels (a, b, and c) were all significantly lower after treatment, and there were no significant differences of control group's CAT, CST, and CV levels before and after treatment. The serum VEGF level (d) in the treatment group was significantly lower after treatment, and there was no significant difference of control group's VEGF before and after treatment. T: treatment; C: control.

cataract or small pupil [29]. The fundus fluorescein angiography (FFA) has no extra requirements for crystalline lens and pupils while the blood pressure, glucose, and liver and kidney functions need to be strictly controlled for using fluorescein sodium contrast agent. However, it was difficult to widely make angiography examinations in this study because the procedure of FFA was more time-consuming and expensive than fundus photograph [30]. In addition, the related study suggested that the sensitivity and accuracy of fundus photography are higher than of FFA combined with fundus photography (sensitivity: 0.63 vs 0.54 ; accuracy: 0.81 vs 0.79 ) [31]. The specificity of fundus photography is comparable with FFA combined with fundus photography (0.93 vs 0.97 ) in diagnosing polypoidal choroidal vasculopathy [31]. Meanwhile, in order to avoid the short-coming of fundus photography, NPDR patients with severe cataract of both eyes and small pupils with high intraocular pressure have been excluded before enrollment. Therefore, the fundus photography was chosen for the NPDR patients in our study. The study results suggested that the remission rate of NPDR severity in the treatment group was significantly higher than that of the control group $(25.4 \%$ vs $9.3 \%, P=0.01)$ and the progression rate of NPDR severity in treatment group was significantly lower than that of the control group $(4.2 \%$ vs $18.7 \%, P=0.007)$ after six months' treatment. It could be concluded that Jiahua tablets in the treatment group played an important role in improving the NPDR degrees because there were no significant differences of risk factors (blood glucose, blood lipid, blood pressure, age, and DM duration) and basic treatments (hypoglycemic, hypotensive, lipid regulation, and antiplatelet agents) between two groups during the study period. Additionally, the study results showed that the adverse reactions were not increased by Jiahua tablets because there were no significant differences of liver and kidney functions between two groups after six months' treatment. The efficacy and safety of Abelmoschus manihot in treating NPDR were preliminarily confirmed.

Vision, acting as the important indicator of DR improvement, was also compared between both groups before and after treatment. In this study, the ETDRS vision chart derived from the "Early Treatment Diabetic Retinopathy Study," is recommended by the "National Eye Institute" and "Food and Drug Administration" of America. It is also considered as a recognized method for assessing visual acuity in clinical trials $[32,33]$. It is more accurate to evaluate the levels of DR vision due to the objective quantification of 


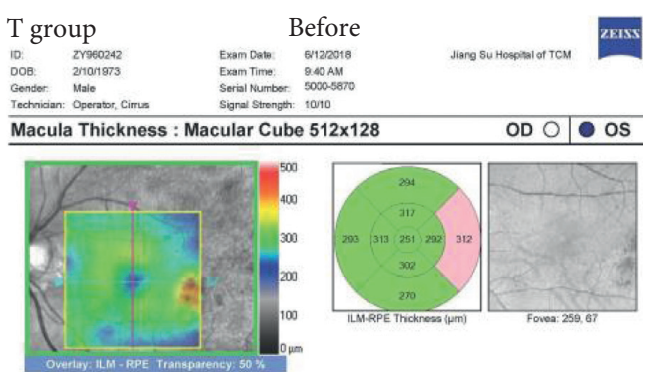

(a)
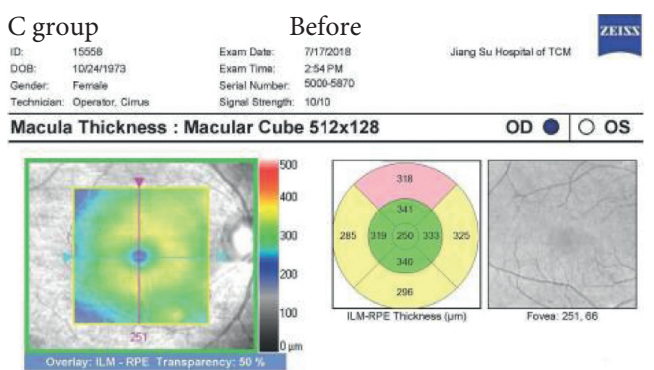

(c)
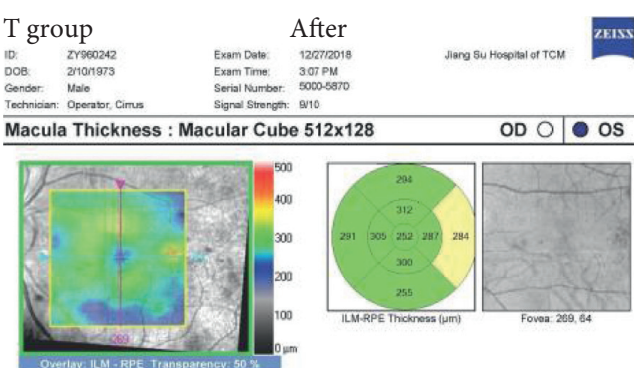

(b)
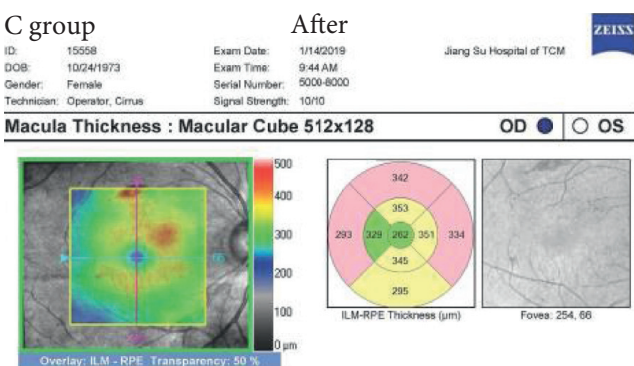

(d)

Figure 4: The typical changes of OCT images and parameters before and after treatment in the two groups. As shown in the figure, the macular thicknesses in the treatment group were significantly lower after six months' treatment. In contrast, the macular thicknesses in the control group were not significantly reduced. T: treatment; C: control.

TABLE 8: Comparisons of serum VEGF levels between two groups before and after treatment.

\begin{tabular}{lcccccc}
\hline & \multicolumn{2}{c}{ T group } & \multicolumn{2}{c}{ C group } & $P_{2}$ \\
& Before $(n=40)$ & After $(n=38)$ & Before $(n=40)$ & After $(n=39)$ & $P_{1}$ \\
\hline VEGF $(\mathrm{ng} / \mathrm{ml})$ & $0.21(0.14,0.58)$ & $0.16(0.10,0.23)$ & $0.21(0.13,0.66)$ & $0.23(0.12,0.64)$ & 1.00 & $0.02^{*}$ \\
\hline
\end{tabular}

T: treatment; C: control; $\mathrm{P}_{1}$ : T group vs $\mathrm{C}$ group before treatment; $\mathrm{P}_{2}$ : T group vs $\mathrm{C}$ group after treatment, ${ }^{*} \mathrm{P} \leq 0.05$.

TAвLE 9: Comparisons of hypoglycemic, hypotensive, and other basic treatment drugs between two groups.

\begin{tabular}{|c|c|c|c|}
\hline & T group $(n=38)$ & C group $(n=39)$ & $P$ \\
\hline \multicolumn{4}{|l|}{ Hypoglycemic drugs } \\
\hline Metformin & 16 & 17 & 0.90 \\
\hline Sulfonylureas & 16 & 12 & 0.30 \\
\hline Glinides & 1 & 0 & 0.49 \\
\hline Acarbose & 18 & 14 & 0.31 \\
\hline Thiazolidinedione & 3 & 3 & 1.00 \\
\hline Insulin & 2 & 0 & 0.24 \\
\hline Insulin-analog & 21 & 23 & 0.74 \\
\hline GLP-1 & 4 & 6 & 0.77 \\
\hline DPP-4 & 12 & 19 & 0.13 \\
\hline SGLT-2 & 3 & 1 & 0.59 \\
\hline \multicolumn{4}{|l|}{ Hypotensive drugs } \\
\hline CCB & 17 & 11 & 0.13 \\
\hline$\beta \mathrm{RB}$ & 7 & 9 & 0.61 \\
\hline ACEI & 2 & 1 & 0.98 \\
\hline $\mathrm{ARB}$ & 8 & 8 & 0.95 \\
\hline Diuretic & 3 & 4 & 1.00 \\
\hline \multicolumn{4}{|l|}{ Other treatments } \\
\hline Aspirin & 7 & 10 & 0.45 \\
\hline Statins & 13 & 19 & 0.20 \\
\hline ARI & 1 & 0 & 0.49 \\
\hline
\end{tabular}

T: treatment; C: control; $\mathrm{CCB}$ : calcium channel blockers; $\beta$ RB: $\beta$ receptor blockers; ACEI: angiotensin-converting enzyme inhibitors; ARB: angiotensin II receptor blockers; ARI: aldose reductase inhibitor. 
severe vision by the ETDRS vision chart [34]. Our present results suggested that there were no significant differences of ETDRS scores between two groups before treatment (73 (67, $79)$ vs $74(68,80)$ points, $P=0.26)$. However, the ETDRS score of the treatment group was significantly higher than that of the control group after six months' treatment (78 (72, $82)$ vs $72(67,80)$ points, $P<0.001)$. Similar to the above discussion about NPDR remission and progression rates, the improvement of NPDR vision in the treatment group was dependent neither of strict control of risk factors such as blood glucose, blood pressure, and blood lipids nor of the difference of basic treatments between two groups. The additional benefit of vision in the treatment group was mainly attributed to oral administration with Abelmoschus manihot.

$\mathrm{DME}$ is the most frequent cause of vision loss in DR. The severity of DME is usually evaluated by OCT which is a noncontact and noninvasive optical imaging technology. There was remarkable development of enhanced depth imaging (EDI) about OCT technology, which has improved the image quality of deeper structures. Swept-source OCT (SS-OCT) especially enables the choroid to be imaged at greater depth and using shorter acquisition times than those required for EDI using spectral-domain OCT (SD-OCT) $[35,36]$. Both OCT devices had comparable repeatability for retinal thickness measurement in normal eyes and eyes with retinal disease [37]. The recent study suggested that diabetic choroidopathy (DC) should be evaluated in clinical trials of drugs targeting DR because vascular changes similar to those in DR are occurring in DC [38]. The preferred evaluation method of choroidal vascular density and volume in DC is SS-OCT because of particularly higher -resolution imaging of the choroid and deeper signal penetration by longer wavelength [39]. However, the relevant study indicated that there was no significant difference of choroidal thickness between NPDR alone and NPDR combined with DME. It also suggested that the notable submacular choroidal pathology might only become present in later stages of DR [39]. Besides, the OCT instrument of Cirrus HD 5000 used in this study is very suitable for clinical research due to the characteristics of convenient operation, stable performance, clear image, automatic request, and vertical comparison [40]. Meanwhile, there was an age-related normal Chinese database for comparison in the instrumental system of Cirrus HD 5000. Therefore, the SD-OCT instrument of Zeiss Cirrus HD 5000 was chosen for the NPDR patients in our study. The present study suggested that there were no significant differences of CAT, CST, and CV in the macular region between two groups before treatment; however, the treatment group's CAT, CST, and CV were significantly lower after six months' treatment (CAT: $286(278,302)$ vs $282(270,295) \mu \mathrm{m}, P<0.0001$; CST: $251(239,274)$ vs 248 $(235,265) \mu \mathrm{m}, P<0.0001$; CV: $10.3(10.0,10.9)$ vs 10.1 (9.7, 10.6) $\left.\mathrm{mm}^{3}, P<0.0001\right)$, while there were no significant differences of control group's CAT, CST, and CV before and after treatment (CAT: $287(279,294)$ vs $287(279,295) \mu \mathrm{m}$, $P=0.27$; CST: $250(240,266)$ vs $252(238,266) \mu \mathrm{m}, P=0.72$; CV: $10.4(10.1,10.6)$ vs $\left.10.4(10.1,10.7) \mathrm{mm}^{3}, P=0.53\right)$. It is concluded that Abelmoschus manihot plays an important role in inhibiting macular edema caused by diabetic retinopathy in this study, although severe DME scarcely occurred in NPDR patients. Our further studies about interfering PDR and severe DME with Abelmoschus manihot have been started.

Previous studies showed that the DR progression could be alleviated by inhibiting the activity of VEGF in the retina because the VEGF upregulation was a key factor leading to the BRB destruction and the pathogenesis of DR [41]. AntiVEGF agent can prevent the development of DR through the mechanism that it specifically combines with VEGF to form an immune complex and loses the ability to bind to VEGF receptor $[42,43]$. It is difficult to assay VEGF levels directly in the retina or vitreous body of DR patients. So, in order to investigate the possible effect of Abelmoschus manihot on VEGF, the serum VEGF levels were assayed by VEGF ELISA kit in this cohort. The data showed that there was no significant difference of serum VEGF levels between two groups before treatment $(0.21(0.14,0.58)$ vs $0.21(0.13,0.66)$ $\mathrm{ng} / \mathrm{ml}, P=1.00)$. There was no significant difference of VEGF levels in the control group before and after treatment $(0.21(0.13,0.66)$ vs $0.23(0.12,0.64) \mathrm{ng} / \mathrm{ml}, P=0.85)$. However, the VEGF levels in the treatment group were significantly improved after six months' treatment $(0.21$ $(0.14,0.58)$ vs $0.16(0.10,0.23) \mathrm{ng} / \mathrm{ml}, P=0.0026)$. It could be concluded that the change of serum VEGF levels were associated with Abelmoschus manihot, and the downregulation of VEGF might play an important role in its therapeutic effect on NPDR. Our future work will design relevant experiments in vivo and in vitro to explore the molecular mechanisms underlying the therapeutic effect of Abelmoschus manihot on DR.

\section{Conclusions}

In summary, the present study offered the first clinical evidence that Abelmoschus Manihot could improve the severity of NPDR, ETDRS vision scores, macular edema, and serum VEGF levels. It could be taken as a novel complementary and alternative strategy for treating type 2 diabetic nonproliferative retinopathy.

\section{Data Availability}

The data used to support the findings of the study are available from the corresponding author upon request.

\section{Conflicts of Interest}

The authors declare that there are no conflicts of interest.

\section{Acknowledgments}

This work was supported by the Innovative Development Funding of Affiliated Hospital of Nanjing University of Chinese Medicine (Y2018CX10), Natural Science Foundation of Jiangsu Province (BK20191090), and National Natural Science Foundation of China (81774248). 


\section{References}

[1] D. S. W. Ting, G. C. M. Cheung, and T. Y. Wong, "Diabetic retinopathy: global prevalence, major risk factors, screening practices and public health challenges: a review," Clinical \& Experimental Ophthalmology, vol. 44, no. 4, pp. 260-277, 2016.

[2] J. W. Y. Yau, S. L. Rogers, R. Kawasaki et al., "Global prevalence and major risk factors of diabetic retinopathy," Diabetes Care, vol. 35, no. 3, pp. 556-564, 2012.

[3] G. S. Tan, A. Gan, C. Sabanayagam et al., "Ethnic differences in the prevalence and risk factors of diabetic retinopathy," Ophthalmology, vol. 125, no. 4, pp. 529-536, 2018.

[4] American Academy of Ophthalmology Retina/Vitreous Panel, Preferred Practice Pattern ${ }^{\circledR}$ Guidelines. Diabetic Retinopathy, American Academy of Ophthalmology, San Francisco, CA, USA, 2017, http://www.aao.org/ppp.

[5] D. Zur, M. Iglicki, and A. Loewenstein, "The role of steroids in the management of diabetic macular edema," Ophthalmic Research, vol. 62, no. 4, pp. 231-236, 2019.

[6] P. Mello Filho, G. Andrade, A. Maia et al., "Effectiveness and safety of intravitreal dexamethasone implant (ozurdex) in patients with diabetic macular edema: a real-world experience," Ophthalmologica, vol. 241, no. 241, pp. 9-16, 2019.

[7] M. Iglicki, D. Zur, C. Busch, M. Okada, and A. Loewenstein, "Progression of diabetic retinopathy severity after treatment with dexamethasone implant: a 24-month cohort study the 'DR-Pro-DEX Study," Acta Diabetologica, vol. 55, no. 6, pp. 541-547, 2018.

[8] M. Iglicki, D. Lingua::EN::Titlecase, D. Zur et al., “TRActional DIabetic reTInal detachment surgery with co-adjuvant intravitreal dexamethasONe implant: the TRADITION STUDY," Acta Diabetologica, vol. 56, no. 10, pp. 1141-1147, 2019.

[9] M. Iglicki, C. Busch, D. Zur et al., "Dexamethasone implant for diabetic macular edema in naive compared with refractory eyes," Retina, vol. 39, no. 1, pp. 44-51, 2019.

[10] F. Semeraro, F. Morescalchi, A. Cancarini et al., "Diabetic retinopathy, a vascular and inflammatory disease: therapeutic implications," Diabetes \& Metabolism, vol. 45, no. 6, pp. 517-527, 2019.

[11] Z. H. Xu, Y. Y. Gao, H. T. Zhang et al., "Progress in experimental and clinical research of the diabetic retinopathy treatment using traditional Chinese medicine," The American Journal of Chinese Medicine, vol. 46, pp. 1421-1447, 2018.

[12] T. Caro-Ordieres, G. Marín-Royo, L. Opazo-Ríos et al., "The coming age of flavonoids in the treatment of diabetic complications," Journal of Clinical Medicine, vol. 9, no. 2, p. E346, 2020.

[13] H.-D. Cai, S.-L. Su, D.-W. Qian et al., "Renal protective effect and action mechanism of Huangkui capsule and its main five flavonoids," Journal of Ethnopharmacology, vol. 206, pp. 152-159, 2017.

[14] L. Shi, L. Feng, M. Zhang et al., "Abelmoschus manihot for diabetic nephropathy: a systematic review and meta-analysis," Evidence-Based Complementary and Alternative Medicine, vol. 2019, Article ID 9679234, 21 pages, 2019.

[15] W. Wu, W. Hu, W. B. Han et al., "Inhibition of akt/mTOR/ p70S6K signaling activity with huangkui capsule alleviates the early glomerular pathological changes in diabetic nephropathy," Frontiers in Pharmacology, vol. 9, p. 443, 2018.

[16] S. Liu, L. Ye, J. Tao, C. Ge, L. Huang, and J. Yu, "Total flavones of Abelmoschus manihot improve diabetic nephropathy by inhibiting the iRhom2/TACE signalling pathway activity in rats," Pharmaceutical Biology, vol. 56, no. 1, pp. 1-11, 2017.
[17] Z. Yang, H. Tang, Q. Shao et al., "Enrichment and purification of the bioactive flavonoids from flower of Abelmoschus manihot (L.) medic using macroporous resins," Molecules, vol. 23, no. 10, 2018.

[18] J. Li, J. Zhang, and M. Wang, "Extraction of flavonoids from the flowers of Abelmoschus manihot (L.) medic by modified supercritical $\mathrm{CO}_{2}$ extraction and determination of antioxidant and anti-adipogenic activity," Molecules, vol. 21, no. 7, 2016.

[19] F. Li, Y. Bai, M. Zhao et al., "Quercetin inhibits vascular endothelial growth factor-induced choroidal and retinal angiogenesis in vitro," Ophthalmic Research, vol. 53, no. 3, pp. 109-116, 2015.

[20] B. Kumar, S. K. Gupta, T. C. Nag et al., "Retinal neuroprotective effects of quercetin in streptozotocin-induced diabetic rats," Experimental Eye Research, vol. 125, pp. 193-202, 2014.

[21] C. S. Kim, J. Kim, Y. S. Kim et al., "Improvement in diabetic retinopathy through protection against retinal apoptosis in spontaneously diabetic torii rats mediated by ethanol extract of osteomeles schwerinae C.K. Schneid," Nutrients, vol. 11, no. 3, 2019.

[22] B. Chen, T. He, Y. Xing, and T Cao, "Effects of quercetin on the expression of MCP-1, MMP-9 and VEGF in rats with diabetic retinopathy," Experimental and Therapeutic Medicine, vol. 14, no. 14, pp. 6022-6026, 2017.

[23] G. Giurdanella, C. D. Anfuso, M. Olivieri et al., "Aflibercept, bevacizumab and ranibizumab prevent glucose-induced damage in human retinal pericytes in vitro, through a PLA2/ COX-2/VEGF-A pathway," Biochemical Pharmacology, vol. 96, no. 3, pp. 278-287, 2015.

[24] Y. Lin, L. Li, J. Liu et al., "SIRT1 deletion impairs retinal endothelial cell migration through downregulation of VEGFA/VEGFR-2 and MMP14," Investigative Opthalmology \& Visual Science, vol. 59, no. 13, pp. 5431-5440, 2018.

[25] M. Li, S. Wang, S. Wang et al., "Occludin downregulation in high glucose is regulated by SSTR2 via the VEGF/NRP1/Akt signaling pathway in RF/6A cells," Experimental and Therapeutic Medicine, vol. 14, no. 2, pp. 1732-1738, 2017.

[26] G. Giurdanella, F. Lazzara, N. Caporarello et al., "Sulodexide prevents activation of the PLA2/COX-2/VEGF inflammatory pathway in human retinal endothelial cells by blocking the effect of AGE/RAGE," Biochemical Pharmacology, vol. 142, pp. 145-154, 2017.

[27] A. Jain, S. Saxena, V. K. Khanna et al., "Status of serum VEGF and ICAM-1 and its association with external limiting membrane and inner segment-outer segment junction disruption in type 2 diabetes mellitus," Molecular Vision, vol. 19, pp. 1760-1768, 2013.

[28] M. Takeyama, M. Yoneda, M. Gosho et al., "Decreased VEGFA and sustained PEDF expression in a human retinal pigment epithelium cell line cultured under hypothermia," Biological Research, vol. 48, p. 42, 2015.

[29] M. M. P. N. Piyasena, J. L. Y. Yip, D. MacLeod et al., "Diagnostic test accuracy of diabetic retinopathy screening by physician graders using a hand-held non-mydriatic retinal camera at a tertiary level medical clinic," BMC Ophthalmology, vol. 19, no. 1, p. 89, 2019.

[30] A. Gaudric, "New retinal imaging techniques," Revue du Praticien, vol. 63, no. 1, pp. 49-54, 2013.

[31] V. Chaikitmongkol, P. Khunsongkiet, D. Patikulsila et al., "Color fundus photography, optical coherence tomography, and fluorescein angiography in diagnosing polypoidal choroidal vasculopathy," American Journal of Ophthalmology, vol. 192, pp. 77-83, 2018. 
[32] P. K. Kaiser, "Prospective evaluation of visual acuity assessment: a comparison of snellen versus ETDRS charts in clinical practice (An AOS Thesis)," Transactions of the American Ophthalmological Society, vol. 107, no. 107, pp. 311-324, 2009.

[33] V. Chaikitmongkol, O. Nanegrungsunk, D. Patikulsila, P. Ruamviboonsuk, and N. M. Bressler, "Repeatability and agreement of visual acuity using the ETDRS number chart, landolt C chart, or ETDRS alphabet chart in eyes with or without sight-threatening diseases," JAMA Ophthalmology, vol. 136, no. 3, pp. 286-290, 2018.

[34] H.-K. Kuo, M.-T. Kuo, I.-S. Tiong, P.-C. Wu, Y.-J. Chen, and C.-H. Chen, "Visual acuity as measured with landolt C chart and early treatment of diabetic retinopathy study (ETDRS) chart," Graefe's Archive for Clinical and Experimental Ophthalmology, vol. 249, no. 4, pp. 601-605, 2011.

[35] M. W. Lee, H. J. Park, Y. I. Shin et al., "Comparison of choroidal thickness measurements using swept source and spectral domain optical coherence tomography in pachychoroid diseases," PLoS One, vol. 15, no. 2, Article ID e0229134, 2020.

[36] C. Mitsch, J. Lammer, S. Karst et al., "Systematic ultrastructural comparison of swept-source and full-depth spectral domain optical coherence tomography imaging of diabetic macular oedema," British Journal of Ophthalmology, vol. 2019, Article ID 314591, 2019.

[37] E. H. Hong, S. J. Ryu, M. H. Kang et al., "Comparison of repeatability of swept-source and spectral-domain optical coherence tomography for measuring inner retinal thickness in retinal disease," PLoS One, vol. 14, no. 1, Article ID e0210729, 2019.

[38] G. A. Lutty, "Diabetic choroidopathy," Vision Research, vol. 139, pp. 161-167, 2017.

[39] J. C. Wang, I. Laíns, J. Providência et al., "Diabetic choroidopathy: choroidal vascular density and volume in diabetic retinopathy with swept-source optical coherence tomography," American Journal of Ophthalmology, vol. 184, pp. 75-83, 2017.

[40] E. Baghdasaryan, X. Huang, K. M. Marion et al., "Reproducibility of central corneal thickness measurements in normal eyes using the Zeiss Cirrus 5000 HD-OCT and pentacam HR," The Open Ophthalmology Journal, vol. 12, no. 1, pp. 72-83, 2018.

[41] E. Obert, R. Strauss, C. Brandon et al., "Targeting the tight junction protein, zonula occludens-1, with the connexin 43 mimetic peptide, aCT1, reduces VEGF-dependent RPE pathophysiology," Journal of Molecular Medicine, vol. 95, no. 5, pp. 535-552, 2017.

[42] B. Bahrami, T. Hong, M. C. Gilles et al., "Anti-VEGF therapy for diabetic eye diseases," Asia-Pacific Journal of Ophthalmology, vol. 6, no. 6, pp. 535-545, 2017.

[43] L. Q. Zhao and J. W. Cheng, "A systematic review and metaanalysis of clinical outcomes of intravitreal anti-VEGF agent treatment immediately after cataract surgery for patients with diabetic retinopathy," Journal of Ophthalmology, vol. 2019, Article ID 2648267, 10 pages, 2019. 